\title{
ROMANIAN AIR FORCE OPERATIONAL CAPABILITIES GENERATION PROCESS
}

\section{Air Fleet General Alexandru-Petru Găluşcă}

\begin{abstract}
The article examines the new multi-role fighter program in the context of the Romanian Air Force operational capabilities generation process and elicits the major requirements to the future platform.
\end{abstract}

Keywords: Decision-making process, modelling, multi-role fighter, advanced air capabilities.

\section{Introduction}

The Romanian Air Force identifies the need for new multi-role aircraft procurement based on assessment of existing capabilities and limitations, as well as force proposals to the Romanian Air Force generated once the country joined the North Atlantic Alliance. This article will present the Romanian experience in analysing the requirements related to the goals that the country undertakes within NATO. Based on this process, The Romanian Air Force was able to define and better understand the fighter configuration and requirements that are necessary, affordable and available, as well as to elaborate a strategic view related to Air Force operational capabilities generation process. The article offers also brief conclusions regarding the Air Force capabilities generation process, the procurement being just one step within that process.

\section{Air Force Capabilities Assessment}

The full integration of Romania in NATO is continuing and the required Air Force capabilities are defined in order to join the components identified at the 2002 NATO Summit in Prague as Defence Capabilities Initiative:

- Deployability and Mobility (getting forces to the crisis quickly);

- Effective engagement (improving force capabilities);

- Consultation, Command and Control (awareness and control);

- Survivability;

- Sustainability and Logistics. 


\section{Romania's existing capability}

Romanian still operates MiG-21 Lancer as the main Air Force vector to comply with national and NATO air defence requirements. Its capabilities are mainly based on the improvements through the upgrade program of the MiG-21 M and MF versions. The upgrade program has been focused mainly to integrate new avionics and weapons system, including:

- Advanced avionics operated through modern HOTAS and glass cockpit;

- Hybrid Navigation System, based on INU/ GPS integration, as well as ILS/ VOR systems;

- Fire Control Radar operating in both air-to-air and air-to-ground modes;

- $\quad$ Short-range infrared missiles capable to engage within visual range (WVR missiles);

- Laser designator pod;

- Laser and IR guided bombs, based on Mk 82 bombs;

- Electronic warfare systems, including flare \& chaff dispensers, radar warning receiver (RWR) and electronic counter measures (ECM) pod;

- VHF/UHF radios;

- Identification Friend or Foe (IFF);

- Video recording system for mission assessment.

The analysis of the Air Defence-Limited (ADI) vs. Air Defence-Advanced (ADX) capabilities and Fighter Bomber-Limited vs. Fighter Bomber-Advanced vs. (FBI vs. FBX) roles of the of the existing MiG-21 platform, related to Air Policing, allows to identify major limitations in:

- Range and endurance;

- Low maneuverability;

- Major pilot load for aircraft handling;

- Number of stores;

- No beyond visual range (BVR) capabilities;

- Lack of air-to-ground missiles;

- Aging platforms and, respectively, considerable logistic and maintenance effort to sustain the aircraft. 


\section{Romanian Current NATO force proposals}

Two Romanian main operating air bases perform air policing missions within the allied air defence system, and four aircraft have been designated as deployable for airto-ground missions. Currently, the aircraft may perform air offensive and defensive operations, with operational capabilities assumed to be ADI and FBI (air defence \& air to ground capabilities - limited). For the future, Romania intends to achieve ADX and FBX capabilities (air defence \& air to ground capabilities - advanced) through procurement of a new multi-role fighter.

To determine configurations that comply with requirements we have built models, serving as a decision support tool. The "Multi-Role Fighter" system (aircraft, logistics support, training, weapons, infrastructure), could be evaluated in comparison with the actual situation by using these analytical models. The main tasks are:

a. to estimate in advance how the system will cope with a given tactical situation based on a given aircraft configuration and available weapons;

b. to evaluate the system needs for development;

c. to compare different types of aircraft.

We define the missions in peace time, crisis and war situation, based on homeland defence scenarios and NATO scenarios. These missions (intercept, close air support, air interdiction, and reconnaissance) define air-to-air and air-to-ground roles. In some more detail, the missions the aircraft should perform are:
Air-to-Air Role
- Intercept
- CAP - Combat Air Patrol
Air-to-Ground Role
- Battlefield Air Interdiction / Air Interdiction
- Anti - Ship Missions
- Suppression of Enemy Air Defence

Reconnaissance Missions

We also developed impact criteria to find how requirements influence the aircraft configuration, as a qualitative description:

L _ Low (desired capabilities, may be replaced using procedures)

M - Medium (desired capabilities, may be supported through procedures; medium performance)

$\mathrm{H} \quad-$ High (highly required, best performance and modes of operation required) 
The output of these models should be Improving Forces Capabilities through Effective Engagement.

A model one can apply is Kill - Live Chain Analysis that evaluates the aircraft/force capability to detect, identify, attack, egress, asses and survive within given tactical situation. Different aircraft systems contribute to a certain level of performances within this model:

Detect $\quad-$ Sensors have an important impact

Identify $\quad-$ A combination of IFF, radar and data link provides best data to avoid fratricide and identify target for mission effectiveness

Attack - A combination of aircraft performance and weapons decide the effectiveness of the aircraft as a weapon

Egress - Performance and an electronic warfare suite will always give the pilot best chances to leave target area fast and safe

Asses $\quad-$ A recording and communications system provides the data for damage assessment as an important phase of the mission planning process

Survive $\quad-$ An electronic warfare suite and performance have an impact on the system's survivability level

\section{Additional Considerations}

In developing its defence capabilities, Romania applies a programmatic approach accounting for national and collective capability requirements, based on the NATO roadmap or decisions, current status and capabilities assessment, and assessment of a long term budgetary framework. Sustainability and logistics, deployability and mobility are among the additional factors with considerable impact on procurement decisions.

\section{Sustainability and logistics}

Acquisition planning is based firstly on defining the "Multi-role Fighter" as a system, including:

1. Aircraft with main and alternate mission equipment;

2. Integrated logistic support, including maintenance, spare parts and equipment, documentation, test equipment, etc.)

3. Training of pilots and technicians;

4. Weapons, including air-to-air missiles, air-to-ground weapons, pods, ammunition, etc.

5. Infrastructure. 
Logistic support should be based on long-term planning to cope both with acquisition process (costs are the main risk factor) and the capability to support operation along the aircraft service life. Systems that are proved in operations may represent a key factor to reduce costs of operation and support, based on other partner experience, mature products, high level of integration of logistics support, commonality, etc.

A realistic estimation of the resources should be performed in the long-term resource assessment, to include estimates of national and defence budgets. This may improve operation and support planning and resource allocation. Also, this estimate is important in order to identify the optimum fighter concept that is appropriate vis a vis national needs, collective security requirements, etc., i.e. deciding on single-engine vs. two-engine solution, tailor the number of required aircraft, allow for logistics support based multi-year programming and statistics based planning, etc.

\section{Deployability and mobility}

High level of deployability is maintained by applying certain measures through the fighter life time, based on the concept design within acquisition's early phases, as well as through long term planning, that address:

1. System reliability;

2. High level of readiness, including personnel, aircrafts, weapons, logistic support and others, generated during peacetime;

3. Availability of strategic/ medium air/sea/ground transportation capabilities;

4. High level of training of the personnel, to allow mission and task allocation at short notice;

5. Standard operating procedures to be used in performing missions and tasks, as well as for command and control.

Several factors defining the aircraft capabilities should be accounted for in assessing the level of deployability. That includes:
a. Air refuelling capability;
b. Communication and identification systems;
c. Data link;
d. Aircraft performance;
e. Navigation system performances;
f. Electronic warfare system;
g. Electronic countermeasures;
h. Night vision. 
The elements above have a degree of influence in mission planning, execution, selfprotection, the capability to deploy to air bases through airspace having a certain level of threat (both air- and ground-based threats), independence related to day/night and all-weather capabilities, and others.

\section{Conclusions}

In selecting a multi-role fighter, it has to be examined as a system that includes the aircraft itself, its weapons, training, logistics support and infrastructure concepts and requirements, as well as the concept and procedures for its operation and maintenance. Decisions on the aircraft configuration should take account of the following main considerations:

- Effectiveness: the capabilities to detect, identify and attack targets both in air-to-air and air-to-ground roles;

- Survivability: the capabilities required to survive when operating in a threatening environment;

- Supportability: the efforts to procure and operate the system should be commensurate to the anticipated economic and financing environment;

- Affordability: the procurement of the system should be compatible to economic and financing situation and the budget available.

Among the additional considerations are:

- Requirements to effectively complete assigned missions;

- Availability: the considered aircraft configurations should be available; in addition, they should be based on the "open configuration" concept in order to reduce further development cost;

- Affordability: a certain level of capabilities has to be acquired given budgetary limitations; when the available budgets are temporarily limited, gradual force capability generation may be the appropriate approach.

Thus, the Romanian capability generation process supports decision-making in view of both capabilities and long-term affordability and selection of the "best available" option.

Air Fleet General ALEXANDRU-PETRU GĂLUŞCĂ, Ph.D., is Chief of Training and Doctrine at the Headquarters of the Romanian Air Force. 